\title{
Institucionalização e reconhecimento da assistência farmacêutica em municípios paranaenses, Brasil
}

\author{
Institutionalization and valuation of Pharmaceutical \\ Assistance in Paraná Counties, Brazil
}

Recebido em: 22/09/2016

Aceito em: $23 / 02 / 2017$
Traudi KLEIN; Suellen Vienscoski SKUPIEN

Universidade Estadual de Ponta Grossa. Avenida General Carlos Cavalcanti $n^{\circ} 4748$,

Uvaranas, Ponta Grossa, PR. CEP 84030-900, Brasil.

E-mail:traudiklein@yahoo.com.br

\section{ABSTRACT}

Pharmaceutical Assistance (PA) has as main objective the medicines, its rational use and appropriate management in the health systems, aiming at the well-being of service users. Therefore, it is essential that it is operationalized and recognized by health managers. The objective of this study is to identify the County Health Plans (CHP) of Paraná State (Brazil) and how PA is inserted in the County Health Secretariats. The study is exploratory, using a documentary and bibliographical review. 26 CHP were identified. It was verified that the professionals involved in most the studied municipalities had restricted knowledge regarding the concepts of PA, limiting itself to the cycle of acquisition, storage and dispensation; few of them expand services to strengthen management and ensure the rational use of medicines by patients. It is concluded that the managers should be oriented and charged to develop the services of PA, integrating the services in a multidisciplinary way and offering quality in the provision of health services.

Keywords: Pharmaceutical Services; Unified Health System; Health Management

\section{RESUMO}

A Assistência Farmacêutica (AF) tem como objetivo principal o medicamento, seu uso racional e a gestão apropriada nos sistemas de saúde, visando ao bem-estar dos usuários dos serviços. Portanto, é essencial que esteja operacionalizada e reconhecida pelos gestores em saúde. O objetivo deste estudo é identificar os Planos Municipais de Saúde (PM) paranaenses e como a AF está inserida nas secretarias de saúde. O estudo tem caráter exploratório, utilizando levantamento documental e bibliográfico. Foram identificados 26 PM. Foi constatado que os profissionais envolvidos na maioria dos municípios pesquisados têm conhecimento restrito quanto aos conceitos de AF, limitando-se ao ciclo de aquisição, armazenamento e dispensação; poucos ampliam os serviços de modo a fortalecer a gestão e garantir o uso racional dos medicamentos pelos pacientes. Foi possível concluir que os gestores devem ser orientados e cobrados a desenvolver os serviços de AF, integrando os serviços de maneira multidisciplinar e oferecendo qualidade na prestação dos serviços em saúde.

Palavras-chave: Assistência Farmacêutica; Sistema Único de Saúde; Gestão em Saúde 


\section{INTRODUÇÃO}

Contar com uma política de Assistência Farmacêutica (AF) é prioridade na Saúde Pública. A compreensão do processo de construção, execução, interrelações e a necessidade de avaliação são de fundamental importância para o entendimento no contexto das políticas nas quais ela está inserida, tais como Política Nacional de Saúde (PNS), Política Nacional de Medicamentos (PNM), Política Nacional de Assistência Farmacêutica (PNAF) entre outras. A perspectiva é da integralidade com estabelecimento de estratégias, parcerias e interfaces com outras políticas setoriais, bem como a participação de diferentes atores e segmentos envolvidos. As ações de caráter multidisciplinar devem ter como objetivo principal o acesso e uso racional de medicamentos essenciais, com enfoque no usuário dos serviços farmacêuticos. Os governos precisam assumir o compromisso de programar as propostas explicitadas nas políticas vigentes e nos relatórios das conferências de saúde e de assistência farmacêutica, pois não é possível falar em efetividade da atenção à saúde sem que a AF cumpra seu papel com qualidade (1-4).

A inclusão das ações de AF nos planos de governo de cada esfera governamental é imprescindível para alcançar os resultados propostos pela PNM, sendo preciso buscar a necessária integração desta com o sistema de saúde, bem como a inclusão nos instrumentos de gestão de forma a produzir resultados eficientes na melhoria dos serviços farmacêuticos $(5,6)$.

A AF é um conjunto de ações voltadas ao medicamento, seu uso racional e gestão dentro do Sistema Único de Saúde (SUS). São medidas voltadas a promoção, proteção e recuperação da saúde, tanto individual como coletiva, com o objetivo final do bem-estar dos usuários dos serviços. Sendo o medicamento o objeto principal da $\mathrm{AF}$, há uma demanda significativa de recursos financeiros do Estado. Dentro desse valor está embutido não somente o preço do medicamento, mas também despesas com utilização inadequada e irracional e ineficácia da gestão logística (p. ex., aquisição, armazenamento e dispensação dos medicamentos) (2,3).

A estruturação da AF no SUS começou de forma tardia e em descompasso entre os componentes técnico (seleção, prescrição, dispensação e uso) e logístico (programação, aquisição, armazenamento e distribuição). Essa reorientação diz respeito ao deslocamento do foco da AF restrito ao componente logístico para incluir a melhora da gestão e a qualidade dos serviços, com a promoção do acesso dos cidadãos aos medicamentos essenciais e seu uso racional, tanto do ponto de vista terapêutico quanto dos recursos públicos. Ou seja: tirar a ênfase do produto para destacar o usuário dos serviços $(4,6)$.

O governo admite e reconhece a necessidade da integralidade das ações de AF de acordo com os princípios e diretrizes do SUS, agregando valor às ações e aos serviços de saúde por meio do desenvolvimento do serviço. Nesse contexto, tem sido trabalhado o fortalecimento da AF em ações que envolvem pesquisa, desenvolvimento e produção de medicamentos e insumos, bem como seleção, programação, aquisição, distribuição, dispensação, garantia da qualidade dos produtos e serviços, acompanhamento e avaliação de utilização, na perspectiva da obtenção de resultados concretos e da melhoria da qualidade de vida da população $(1,7)$. Porém, o grande problema brasileiro é que o Ministério da Saúde não especifica prazos para os serviços e há uma grande lacuna de conhecimento do papel e das funções dos farmacêuticos, por ser uma nova lógica implantada no SUS, devido à deficiência de formação específica e falta de orientação adequada aos profissionais. Isso implica dificuldades de implantação dos serviços e consequente atraso no atendimento efetivo à população.

A produção de conhecimento é considerada estratégica para o desenvolvimento da AF bem como dos recursos humanos e serviços. Exige articulação permanente com áreas técnicas, administrativas, coordenação de programas estratégicos de saúde, área administrativo-financeira, planejamento, material e patrimônio, licitação, auditoria, Ministério Público, órgãos de controles, conselhos e profissionais de saúde, entidades de classe, universidades, fornecedores e setores de comunicação da secretaria entre outros segmentos da sociedade, para melhor execução, divulgação e apoio às ações $(1,7)$.

No entanto, pode ser observado que frequentemente a AF não tem o adequado reconhecimento ou a aceitação por outras áreas ou departamentos das Secretarias de Saúde e frequentemente não consta formalmente na estrutura organizacional. Muitas vezes não está inserida no PMS nem no orçamento anual dos municípios, e as ações de organização não estão integradas no SUS, não havendo planejamento nas diferentes etapas do ciclo $(4,8)$.

A estrutura da AF deve respeitar as características de cada município, como perfil epidemiológico, geográfico, social e econômico, sobretudo aquelas relacionadas à saúde. A implantação deve prever: formalização da 
estrutura organizacional com definição das responsabilidades e atribuições dos profissionais envolvidos, infraestrutura adequada que possibilite o desenvolvimento das atividades do ciclo da AF em quantidade e qualidade suficiente para atender ao serviço e recursos humanos com profissionais preparados, capacitados e com atribuições claramente definidas $(1,7,9)$.

A estruturação da AF deve estar prevista nos PMS e no organograma administrativo da gestão. O PMS é um documento que sistematiza o conjunto de proposições políticas do governo municipal na área de saúde. Dessa forma, traduz-se em um instrumento que apresenta as intenções e os resultados a serem buscados no período de quatro anos, com objetivos, diretrizes e metas. Cada município tem autonomia para definir as linhas gerais do processo de elaboração do PMS, respeitando os princípios e diretrizes da legislação e normas complementares do SUS $(10,11)$.

A organização da AF depende de um financiamento sustentado e mantido em qualquer uma das instâncias gestoras do SUS, e deverão ser assegurados os recursos financeiros que viabilizem as ações e sua continuidade. A gestão de pessoas também é parte imprescindível para os gestores, sendo necessário manter um canal de comunicação permanente e incentivar a participação coletiva, inclusive nas decisões, influenciando positivamente a realização de tarefas pelos profissionais $(2,12)$.

É importante lembrar que a AF é multidisciplinar; porém, o farmacêutico, por ser legalmente responsável pelo medicamento, é o profissional competente para o desenvolvimento de atividades como seleção, programação, armazenamento, distribuição e dispensação de medicamentos. Também é estratégica a participação do farmacêutico no processo de aquisição de medicamentos, em especial na elaboração das especificações, estabelecimento de critérios técnicos e emissão de parecer no julgamento das propostas (1-3,9).

O acompanhamento e a avaliação continuada das ações de AF é outra estratégia para solidificar o trabalho. A implantação de sistemas de informação gerenciais auxilia a formulação de políticas de medicamentos e subsidia a tomada de decisões dos gestores. É necessário um sistema de informações que reflita a realidade dos serviços disponibilizando indicadores seletivos e específicos que auxiliem na qualificação do processo de decisão e na racionalização da aplicação dos recursos $(2,3)$.

É sabida a necessidade de aperfeiçoar o uso dos recursos (financeiros, humanos etc.) públicos; e, para tanto, a gestão pública e seu planejamento são funda- mentais para pensar a realidade e agir sobre ela. Considerando que a AF demanda altos investimentos financeiros e capacitação dos profissionais de gestão, é interessante observar se os municípios estão inserindo as atividades farmacêuticas na agenda municipal e se esses serviços estão tendo o devido reconhecimento por parte dos gestores.

Portanto, o objetivo deste estudo é identificar nos PMS como a AF está inserida nas Secretarias Municipais de Saúde do Paraná.

\section{MATERIAL E MÉTODOS}

O estudo tem caráter exploratório, utilizando levantamento documental e consulta bibliográfica.

Os documentos pesquisados e analisados são os PMS do Paraná, que se encontravam disponíveis online para consulta, e também os enviados por e-mail pelas Secretarias de Saúde Municipais a pedido da pesquisadora. O site do governo do Paraná (www.cidadao.pr.gov. br) e o Portal dos Municípios (www.municipios.pr.gov. br) foram consultados, sendo 399 municípios paranaenses pesquisados. Nas informações coletadas nos PMS, pode haver divergência com a realidade local de cada município, que não foi investigada pessoalmente com a equipe de saúde de cada localidade.

O Plano Estadual de Saúde 2012-2015 do Paraná (13) está disponível online e serve como documento norteador das Secretarias Municipais de Saúde do Estado. Os gestores municipais devem levar em consideração a realidade de municípios e regiões, consultando os indicadores sociodemográficos para o estabelecimento e construção de PMS e políticas de enfrentamento dos problemas em saúde, na perspectiva de instrumentalizar o acompanhamento e a avaliação da implantação das políticas no município $(1,2,7,9,11)$.

A análise documental refletiu a inserção da AF nos PMS, o quantitativo de farmacêuticos trabalhando nos municípios estudados e possíveis sugestões de ações de inserção e reconhecimento para os farmacêuticos que trabalham no SUS.

Ao analisar os documentos, os autores responderam às seguintes perguntas, sendo organizadas as respostas em forma de tabela.

1. Qual o número de habitantes do município?

2. Qual o número de profissionais farmacêuticos e quais os serviços desempenhados por profissional?

3. Os profissionais aplicam os conhecimentos de AF na gestão farmacêutica do município? 
4. O município tem equipe(s) de NASF? Há participação do profissional farmacêutico na(s) equipe(s)?

5. O município possui CAF? Há profissional farmacêutico no serviço?

A consulta bibliográfica da AF, definições, obrigações e deveres do farmacêutico, legislação específica do assunto e outros assuntos relacionados foi realizada em bases de dados como Scielo, Lilacs e Periódicos Capes. Em especial, foi consultada a legislação farmacêutica na página da Agência Nacional de Vigilância Sanitária (Anvisa), entre outros, dando preferência a publicações recentes, datadas após 2005 .

\section{RESULTADOS E DISCUSSÃO}

No total foram consultados 26 PMS de municípios paranaenses não restringidos por regiões e sim pela disponibilidade do documento online das prefeituras ou enviado por e-mail por funcionário da Saúde. Os PMS consultados foram referentes às seguintes cidades: Almirante Tamandaré, Antonina, Arapoti, Balsa Nova, Califórnia, Cambará, Campina do Simão, Campo Magro, Campo Mourão, Carambeí, Cascavel, Curitiba, Ibiporã, Jacarezinho, Jaguariaíva, Lapa, Laranjeiras do Sul, Londrina, Maringá, Pinhais, Ponta Grossa, Rebouças, Santa Fé, São José dos Pinhais, Tupãssi e Vera Cruz do Oeste (Tabela 1).

Na pesquisa online, dos 399 municípios paranaenses, somente 16 tinham PMS disponíveis para consulta pública. Destes, 8 eram de municípios com mais de 100.000 habitantes, 1 de município de 50.000 a 100.000 habitantes e 7 de municípios com menos de 50.000 habitantes (Tabela 1). Esses dados remetem ao fato da falta de capacitação e profissionalização dos profissionais envolvidos na gestão do SUS e talvez a falta de demanda pela população para conhecer o documento e exigir que esteja disponível para consulta.

Apesar do contato por email, somente 10 municípios com menos de 50.000 habitantes enviaram o documento (Tabela 1). O resultado revelou que a demanda por informações nos municípios não é atendida via web. Atualmente, com a facilidade de acesso em qualquer ambiente online, entre as proposições para que isso esteja ocorrendo está a falta de capacitação dos envolvidos e de atualização das informações nos sites.

O gestor deve definir e explicitar a organização e o funcionamento da gestão do SUS, considerando a AF no organograma de funcionamento. Em um processo de descentralização de poderes, definir responsáveis para cada etapa e ciclo de trabalho e se preocupar com a profissionalização dos trabalhadores envolvidos; considerando o cenário demográfico e epidemiológico, apresentar os resultados desejados para a sociedade dentro daquele período; e, para que os resultados para a sociedade sejam alcançados, o gestor deve definir perspectivas de trabalho tanto de processos, gestão e financeira e propor ações para operacionalização dos trabalhos $(1,2,7,9)$.

A AF representa atividade de grande impacto financeiro no âmbito do SUS em razão da crescente demanda por medicamentos. Por isso é importante que os gestores considerem e proponham ações com objetivos definidos para a gestão e operacionalização da $\mathrm{AF}$ nos municípios, otimizando os escassos recursos destinados à saúde $(1,2,6,7,9)$.

A atuação específica do farmacêutico, como responsável técnico nos municípios, engloba as unidades de dispensação de medicamentos (farmácia), assistência farmacêutica, vigilância em saúde e laboratórios de análises clínicas. Durante o processo de municipalização da AF, os municípios devem assumir o compromisso de estruturar essa área para desenvolver atividades que lhe são pertinentes. Trata-se de uma atividade multidisciplinar, cuja coordenação deve estar sob responsabilidade do farmacêutico, que tem a formação acadêmica compatível e a atribuição legal para exercê-la $(4,6,14)$.

O Conselho Federal de Farmácia (CFF) propôs, para a estruturação da AF municipal, a presença de uma quantidade mínima de farmacêuticos exercendo funções nas SMS (Tabela 2) (14). Contudo, informação sobre a quantidade de farmacêuticos trabalhando nos municípios estudados mostrou-se incompleta (Tabela 1), e, quando houve disponibilização da informação, a definição das atividades desenvolvidas pelos profissionais não era clara.

Com o avanço das discussões sobre políticas públicas na área da saúde e a formação dos farmacêuticos direcionada para o trabalho no sistema público, os profissionais poderão ser reconhecidos e atuarão cumprindo seu papel na sociedade brasileira (15).

O Ministério da Saúde publicou o manual "Planejar é preciso", no qual são apresentadas informações sobre a organização da AF e sugeridos indicadores de avaliação da qualidade da assistência farmacêutica no SUS, auxiliando os gestores municipais no desenvolvimento das ações de AF (1).

Pode ser percebido que $57 \%$ dos PMS pesquisados (Tabela 1) revelaram conhecimento restrito dos conceitos atuais de AF, limitando-se muitas vezes aos 
processos de aquisição, armazenamento e dispensação de medicamentos ou à rara aplicação de tais conceitos no planejamento. Isso se deve à falta de profissionalização dos gestores, que muitas vezes não são profissionais da saúde, ocupando apenas cargos administrativos comissionados. Além disso, a legislação atual não exige a profissionalização dos gestores, que acabam por não demandar esse conhecimento e capacitação da equipe de Saúde do município.

É importante ressaltar que a AF deve ter caráter sistêmico e multidisciplinar, atuando nas diversas equipes multiprofissionais da equipe de saúde.

Tabela 1: Resultados da pesquisa sobre a Assistência Farmacêutica nos Planos Municipais de Saúde (PMS) de municípios paranaenses*

\begin{tabular}{|c|c|c|c|c|c|c|}
\hline \multicolumn{7}{|c|}{ Informações sobre Planos Municipais de Saúde disponiveis online } \\
\hline Município & habitantes & farmacêuticos & $\begin{array}{c}\text { Aplica conhecimentos } \\
\text { de AF? }\end{array}$ & Possui NASF? & Possui CAF? & Referência \\
\hline $\begin{array}{l}\text { Almirante } \\
\text { Tamandaré }\end{array}$ & 103204 & $\begin{array}{c}05 \\
\text { VISA local } \\
\text { coordenada por } \\
\text { farmacêutico. }\end{array}$ & Sim. & $\begin{array}{l}\text { Planejamento de } \\
\text { implantar. }\end{array}$ & Sim. & $(22)$ \\
\hline Arapoti & 25855 & $\begin{array}{c}05.03 \text { atendendo } \\
\text { o SUS. }\end{array}$ & $\begin{array}{l}\text { Sim, com aplicação } \\
\text { limitada. }\end{array}$ & $\begin{array}{l}\text { Planejamento de } \\
\text { implantar. }\end{array}$ & $\begin{array}{l}\text { Sim, porém } \\
\text { com instalações } \\
\text { e transporte } \\
\text { inadequados. }\end{array}$ & $(23)$ \\
\hline $\begin{array}{l}\text { Campina do } \\
\text { Simão }\end{array}$ & 4076 & 01 & $\begin{array}{l}\text { Sim, com aplicação } \\
\text { limitada. }\end{array}$ & $\begin{array}{c}\text { Planejamento de } \\
\text { implantar em } 2014 .\end{array}$ & Não. & $(24)$ \\
\hline $\begin{array}{l}\text { Campo } \\
\text { Magro }\end{array}$ & 24843 & Sem informação. & $\begin{array}{l}\text { Sim, com aplicação } \\
\text { limitada. }\end{array}$ & $\begin{array}{l}\text { Sim, sem farmacêutico. } \\
\text { Planejamento de } \\
\text { implantar novos } \\
\text { serviços. }\end{array}$ & Não. & $(25)$ \\
\hline $\begin{array}{l}\text { Campo } \\
\text { Mourão }\end{array}$ & 88209 & 19 & $\begin{array}{l}\text { Sim. } \\
\text { Planejamento de } \\
\text { reestruturação dos } \\
\text { serviços. }\end{array}$ & $\begin{array}{l}\text { Sim. } \\
\text { Não especifica os } \\
\text { profissionais que } \\
\text { participam. }\end{array}$ & $\begin{array}{l}\text { Não. } \\
\text { Planejamento de } \\
\text { criar e estruturar } \\
\text { a CAF. }\end{array}$ & $(26)$ \\
\hline Cascavel & 286205 & Sem informação. & Sim. & $\begin{array}{l}\text { Planejamento de } \\
\text { implantar com } \\
\text { participação de } \\
\text { farmacêutico. }\end{array}$ & Sim. & $(27)$ \\
\hline Curitiba & 1751907 & $\begin{array}{l}\text { Sem informação. } \\
\text { Número insuficiente, } \\
\text { alta rotatividade dos } \\
\text { profissionais nas UBS. }\end{array}$ & $\begin{array}{l}\text { Sim, com ausência de } \\
\text { centralização da gestão } \\
\text { e qualidade limitada } \\
\text { das ações em AF. }\end{array}$ & $\begin{array}{l}\text { Sim, com cobertura } \\
\text { insuficiente. } \\
\text { Planejamento de } \\
\text { ampliar o número de } \\
\text { equipes. }\end{array}$ & Não. & $(28)$ \\
\hline Ibiporã & 49111 & $\begin{array}{l}03 \text { profissionais. } \\
\text { VISA local com } \\
\text { participação do } \\
\text { farmacêutico. }\end{array}$ & Sim. & $\begin{array}{l}\text { Sim, com participação } \\
\text { do farmacêutico. }\end{array}$ & $\begin{array}{l}\text { Sim, para } \\
\text { medicamentos } \\
\text { psicotrópicos e } \\
\text { de programas do } \\
\text { Governo. }\end{array}$ & $(29)$ \\
\hline Jacarezinho & 39121 & $\begin{array}{l}\text { 04/03 atendendo } \\
\text { SUS. }\end{array}$ & $\begin{array}{c}\text { Sim, com planejamento } \\
\text { de fortalecer e } \\
\text { qualificar a gestão. }\end{array}$ & $\begin{array}{l}\text { Sim, com } \\
\text { planejamento de } \\
\text { ampliar o serviço. }\end{array}$ & Sem informação. & $(30)$ \\
\hline Lapa & 44932 & Sem informação. & $\begin{array}{l}\text { Sim, com aplicação } \\
\text { limitada. }\end{array}$ & $\begin{array}{l}\text { Planejamento de } \\
\text { implantar em } 2014 .\end{array}$ & $\begin{array}{l}\text { Não. } \\
\text { Planejamento de } \\
\text { criar e estruturar } \\
\text { a CAF. }\end{array}$ & (31) \\
\hline Londrina & 506701 & $\begin{array}{c}\text { 176/150 atendendo } \\
\text { SUS. }\end{array}$ & Sim. & $\begin{array}{l}\text { Sim, com participação } \\
\text { do farmacêutico. }\end{array}$ & Sim. & (32) \\
\hline Maringá & 367410 & $\begin{array}{l}\text { Sem informação. } \\
\text { Número insuficiente } \\
\text { de farmacêuticos. }\end{array}$ & Sim. & Sim. & Sim. & (33) \\
\hline Pinhais & 117008 & 06 & $\begin{array}{l}\text { Sim, com aplicação } \\
\text { limitada. }\end{array}$ & Sim. & Sim. & $(34)$ \\
\hline $\begin{array}{l}\text { Ponta } \\
\text { Grossa }\end{array}$ & 311611 & Sem informação. & $\begin{array}{l}\text { Sim, com aplicação } \\
\text { limitada. }\end{array}$ & $\begin{array}{l}\text { Planejamento de } \\
\text { implantar. }\end{array}$ & Sim. & $(35)$ \\
\hline $\begin{array}{l}\text { São José } \\
\text { dos Pinhais }\end{array}$ & 264210 & Sem informação. & $\begin{array}{l}\text { Planejamento de } \\
\text { estruturar o serviço. }\end{array}$ & $\begin{array}{l}\text { Planejamento de } \\
\text { implantar. }\end{array}$ & Não. & $(36)$ \\
\hline Tupãssi & 7997 & 01 & Sem informação. & Sem informação. & Sem informação. & $(37)$ \\
\hline
\end{tabular}




\begin{tabular}{|c|c|c|c|c|c|c|}
\hline \multicolumn{7}{|c|}{ Informações sobre Planos Municipais de Saúde recebidas por email } \\
\hline Município & habitantes & farmacêuticos & $\begin{array}{l}\text { Aplica conhecimentos } \\
\text { de AF? }\end{array}$ & Possui NASF? & Possui CAF? & Referência \\
\hline Antonina & 17581 & Sem informação. & $\begin{array}{l}\text { Planejamento de } \\
\text { estruturar o serviço. }\end{array}$ & Sem informação. & Sem informação. & $(38)$ \\
\hline Balsa Nova & 11252 & 02 & $\begin{array}{l}\text { Sim, com aplicação } \\
\text { limitada. }\end{array}$ & $\begin{array}{l}\text { Planejamento de } \\
\text { implantar. }\end{array}$ & Sem informação. & $(39)$ \\
\hline Califórnia & 8423 & 02 & $\begin{array}{l}\text { Sim, com planejamento } \\
\text { de fortalecer e } \\
\text { qualificar a gestão. }\end{array}$ & $\begin{array}{l}\text { Planejamento de } \\
\text { implantar. }\end{array}$ & Sem informação. & $(40)$ \\
\hline Cambará & 23871 & Sem informação. & $\begin{array}{l}\text { Sim, com planejamento } \\
\text { de fortalecer e } \\
\text { qualificar a gestão. }\end{array}$ & $\begin{array}{l}\text { Planejamento de } \\
\text { implantar. }\end{array}$ & $\begin{array}{l}\text { Planejamento de } \\
\text { implantar. }\end{array}$ & $(41)$ \\
\hline Carambeí & 20863 & 02 & $\begin{array}{l}\text { Sim, com aplicação } \\
\text { limitada. }\end{array}$ & Sem informação. & Sem informação. & (42) \\
\hline Jaguariaiva & 32882 & 01 & $\begin{array}{l}\text { Sim, com aplicação } \\
\text { limitada. }\end{array}$ & $\begin{array}{l}\text { Planejamento de } \\
\text { implantar. }\end{array}$ & Sem informação. & (43) \\
\hline $\begin{array}{l}\text { Laranjeiras } \\
\text { do Sul }\end{array}$ & 30777 & 04 & $\begin{array}{l}\text { Sim, com aplicação } \\
\text { limitada. }\end{array}$ & Sim. & $\begin{array}{l}\text { Planejamento de } \\
\text { implantar. }\end{array}$ & (44) \\
\hline Rebouças & 14254 & 02 & $\begin{array}{l}\text { Sim, com planejamento } \\
\text { de fortalecer e } \\
\text { qualificar a gestão. }\end{array}$ & Sim. & Sem informação. & $(45)$ \\
\hline Santa Fé & 10432 & 02 & $\begin{array}{c}\text { Sim, com planejamento } \\
\text { de fortalecer e } \\
\text { qualificar a gestão. }\end{array}$ & $\begin{array}{l}\text { Planejamento de } \\
\text { implantar. }\end{array}$ & Sem informação. & (46) \\
\hline $\begin{array}{l}\text { Vera Cruz do } \\
\text { Oeste }\end{array}$ & 8973 & 05 & $\begin{array}{l}\text { Sim, com aplicação } \\
\text { limitada. }\end{array}$ & Sim. & Sem informação. & $(47)$ \\
\hline
\end{tabular}

*OBS: informações coletadas nos PMS, portanto, se há alguma divergência com a realidade local de cada município, esta não foi investigada pessoalmente com a equipe de saúde de cada localidade.

PMS: Plano Municipal de Saúde. AF: Assistência Farmacêutica. NASF: Núcleo de Apoio a Saúde da Família. CAF: Central de Abastecimento Farmacêutico. SMS: Secretaria Municipal de Saúde. SUS: Sistema Único de Saúde. UBS: Unidade Básica de Saúde. VISA: Vigilância Sanitária.

Para melhor compreensão e aplicação dos conhecimentos de AF, o Ministério da Saúde propôs, em 2007, a criação de Núcleos de Apoio à Família (NASF). É uma iniciativa que tem ampliado o número de componentes vinculados às Equipes de Saúde da Família (ESF), reunindo diversos profissionais da área de saúde, dentre eles o farmacêutico, qualificando e potencializando o trabalho, além de permitir a participação em diversas áreas fora do campo de atuação individual da farmácia. Qualquer município brasileiro, desde que tenha ao menos uma Equipe de Saúde da Família, pode implantar equipes NASF. O que vai variar de um município para o outro, conforme o número de equipes de SF que tiver, é a modalidade de NASF a ser implantada, sendo que o farmacêutico faz parte dos profissionais que podem compor as equipes, que é definida pelos gestores municipais. Além disso, a ESF e deve considerar os critérios de prioridade identificados a partir dos dados epidemiológicos, das necessidades do território e das equipes de saúde que serão apoiadas $(14,16)$.

A inserção do farmacêutico no NASF é importante por possibilitar o maior acesso da população ao medicamento e contribuir para o uso racional. O farmacêutico, no NASF, deve desenvolver ações de AF na gestão do medicamento e na assistência à saúde, colaborando com o uso racional de medicamentos pelos pacientes. Entretanto, a proposta do NASF para os farmacêuticos carece ainda de objetivos claros, impedindo o planejamento (por não saber onde se quer chegar) e, consequentemente, a definição do processo de trabalho, que é percebido também para outros profissionais $(14,16)$.

Dos municípios estudados, apenas 11tinham equipes de NASF criadas e atuantes (Tabela 1). Esse processo de implantação e contratualização das equipes já deveria ter acontecido, mas o Ministério da Saúde não especifica prazos para que aconteça. Isso pode ser decorrente da falta de conhecimento do papel e das funções dos farmacêuticos, por ser uma nova lógica implantada no SUS, devido à deficiência de formação específica e falta de orientação adequada aos profissionais ao se iniciarem no NASF (16).

Como proposta para organização da AF nos municípios, o CFF sugeriu que o município possua local apropriado para armazenamento e controle de estoque de produtos farmacêuticos e denomina esse local de CAF. Deve ainda propiciar aos profissionais de saúde o conhecimento da relação atualizada de medicamentos disponíveis. O local deve estar sob responsabilidade técnica do farmacêutico (14). 
Tabela 2: Quantidade mínima de farmacêuticos por número de habitantes municipais e suas funções no município (14).

\begin{tabular}{|c|c|c|}
\hline habitantes & farmacêuticos/habitante & Função do farmacêutico \\
\hline Até 15.000 & Mínimo 01 & $\begin{array}{l}\text { Coordenador da AF } \\
\text { RT pela UD } \\
\text { Carga horária mínima de quatro horas diárias }\end{array}$ \\
\hline $15.001-50.000$ & Mínimo 02, sendo 01 para cada 10.000 hab. & $\begin{array}{l}\text { Coordenador da AF } \\
\text { RT pela UD } \\
\text { RT da CAF }\end{array}$ \\
\hline $50.001-100.000$ & $\begin{array}{l}01 \text { para cada } 10.000 \text { hab, respeitando a regra de } 01 \text { profissional para } \\
\text { cada serviço. }\end{array}$ & $\begin{array}{l}\text { Coordenação da AF } \\
\text { RT pela UD } \\
\text { RT da CAF } \\
\text { RT farmácias de Unidades de Saúde } \\
\text { RT dos Centros de Atenção Psicossocial }\end{array}$ \\
\hline Acima de 100.000 & $\begin{array}{l}01 \text { para cada } 10.000 \text { hab, respeitando a regra de } 01 \text { profissional para } \\
\text { cada serviço. } \\
\text { Respeitando ainda, } 01 \text { para cada } 10.000 \text { hab }\end{array}$ & $\begin{array}{l}\text { Coordenação da AF } \\
\text { RT pela UD } \\
\text { RT da CAF } \\
\text { RT na farmácia ambulatorial } \\
\text { RT na farmácia hospitalar }\end{array}$ \\
\hline
\end{tabular}

AF: Assistência Farmacêutica; UD: Unidade de Dispensação; CAF: Central de Abastecimento Farmacêutico; RT: Responsável Técnico.

A CAF deve contar com uma estrutura física e layout que atendam às necessidades de fluxo inerentes ao serviço, ou seja, espaço condizente com o perfeito recebimento, armazenamento e expedição dos medicamentos e insumos de acordo com a legislação sanitária pertinente. Os parâmetros de segregação de materiais e medicamentos, localização, insolação, ventilação, segurança são estabelecidos em legislação específica $(1,7,17)$.Entre os objetivos principais em manter uma CAF podem ser citados: controlar entrada e saída de medicamentos, tanto no âmbito físico como financeiro, e manter o estoque regular sem faltas. Desse modo diminuindo o número de itens em falta para três medicamentos por mês por um período máximo de 15 dias, não faltaria nenhum medicamento crítico (AAS, soro, paracetamol, omeprazol, captopril entre outros) e controlar a validade, dispensar medicamentos fracionados e identificados com validade e lote $(9,17)$.

De acordo com a pesquisa nos PMS, os 18 municípios estudados ainda não tinham $\mathrm{CAF}$, limitando o armazenamento dos produtos farmacêuticos em unidades de dispensação. Isso pode comprometer a gestão dos produtos farmacêuticos (aquisição, distribuição e dispensação) e a qualidade da AF, levando a gastos desnecessários com perdas de produtos e ainda à falta de medicamentos essenciais para a população. Também pode comprometer a qualidade desses produtos devido à exposição inadequada a fatores climáticos (17).

No Brasil, além de haver falhas na disponibilidade de medicamentos, há dificuldades econômicas dos usuários e ausência de comunicação entre as áreas da saúde. Apesar das mudanças nas políticas de assistência à saúde e de $\mathrm{AF}$, os resultados apontam para a necessida- de de atualização do serviço, tornando-o mais acessível, resolutivo e articulado aos princípios e diretrizes do SUS (18).

Um estudo realizado para avaliar as condições da AF em Parnamirim, RN mostrou a falta de profissionais farmacêuticos e a necessidade de planejamento e melhorias a fim de desenvolver um serviço de qualidade e capaz de atender às necessidades da população (19). Em Guamá, PA, foi constatada a necessidade, principalmente, de aperfeiçoar os processos de acesso aos medicamentos e a discussão sobre o papel do farmacêutico na atenção em saúde, em especial nos novos conceitos de gestão técnica da $\mathrm{AF}$ e gestão clínica do medicamento (20). Em Formosa do Sul, SC, os autores observaram que os profissionais da ESF e os usuários, apesar de considerarem o serviço importante, tinham pouco conhecimento sobre seu conceito e atribuições, reduzido à dispensação de medicamentos feita pelo profissional farmacêutico. Um destaque deste estudo dizia respeito aos profissionais da ESF transferirem aos usuários a responsabilidade pela falta de entendimento sobre o tratamento (21).

\section{CONCLUSÃO}

Ainda que o governo, nas várias instâncias de poder, reconheça a AF como parte importante e imprescindível para o sucesso do SUS, pode ser observado que os gestores locais e os profissionais farmacêuticos muitas vezes não reconhecem a dimensão do trabalho farmacêutico, e sua importância para a população, na prestação de serviços de orientação sobre o uso racional de medicamentos e na gestão da AF. A profissionalização 
não deve vir apenas dos gestores, mas também dos profissionais envolvidos, responsáveis pelo uso de grande parte dos recursos financeiros da saúde dos municípios.

Muitos são os problemas a serem superados no acesso e uso racional de medicamentos. São necessários grandes esforços para melhorar a gestão dos municípios paranaenses no sentido de garantir uma AF plena com uso eficiente dos recursos financeiros para aquisição de medicamentos, sustentabilidade do SUS e melhoria da qualidade de vida dos pacientes.

\section{REFERÊNCIAS}

1. BRASIL. Ministério da Saúde. Secretaria de Ciência, Tecnologia e Insumos Estratégicos. Planejar é preciso: uma proposta de método para aplicação à assistência farmacêutica. $1^{\text {a }}$ ed. Brasília: Editora do Ministério da Saúde; 2006.

2. BRASIL. Conselho Nacional de Secretários de Saúde. Assistência Farmacêutica no SUS. $1^{\mathrm{a}}$ ed. Brasília: CONASS; 2007.

3. SES-PR. Secretaria de Saúde. Assistência Farmacêutica. 2016. Disponível em: http://www.saude.pr.gov.br/modules/conteudo/conteudo.php?conteudo=3059. Acesso em 12 de julho de 2016.

4. Vieira FS. Qualificação dos serviços farmacêuticos no Brasil: aspectos inconclusos da agenda do Sistema Único de Saúde. Rev Panam Salud Publica. 2008; 24(2): 91-100.

5. BRASIL. Ministério da Saúde. Portaria $\mathrm{n}^{\circ}$ 3.916, de 30 de outubro de 1998. Aprovar a Política Nacional de Medicamentos. Diário Oficial da União no $215-\mathrm{E}, 10$ de novembro de 1998. Seção 1. p 18-22.

6. Bruns SF, Luiza VL, Oliveira EA. Gestão da assistência farmacêutica em municípios do estado da Paraíba (PB): olhando a aplicação de recursos públicos. Rev. Adm. Pública. 2014; 48(3): 745-765. DOI: 10.1590/003476121502

7. BRASIL. Ministério da Saúde. Secretaria de Ciência, Tecnologia e Insumos Estratégicos. Assistência farmacêutica na atenção básica: instruções técnicas para sua organização. $2^{\mathrm{a}}$ ed. Brasília: Editora do Ministério da Saúde; 2006b.

8. Vieira FS. Avanços e desafios do planejamento no Sistema Único de Saúde. Ciência Saúde Col. 2009; 14(1): 1565-1577. DOI: 10.1590/S1413-81232009000800030

9. BRASIL. Conselho Regional de Farmácia do Estado de São Paulo. Assistência Farmacêutica Municipal: Diretrizes para Estruturação e Processos de Organização. São Paulo; 2013.

10. BAHIA. Secretaria de Saúde do Estado. Manual prático de apoio a elaboração de Planos Municipais de Saúde. Salvador: SESAB; 2009.

11. Kleba ME, Comerlatto C, Frozza KM. Instrumentos e mecanismos de gestão: contribuições ao processo decisório em conselhos de políticas públicas. Rev. Adm. Pública. 2015; 49(4): 1059-1079. DOI: 10.1590/00347612125666
12. Vieira FS, Zucchi P. Financiamento da Assistência Farmacêutica no Sistema Único de Saúde. Saúde Soc. São Paulo. 2013; 22(1): 73-84. DOI: 10.1590/S010412902013000100008

13. SES-PR Secretaria de Estado da Saúde do Paraná. Plano Estadual de Saúde Paraná 2012-2015. Curitiba: SESA Secretaria de Estado da Saúde do Paraná, 2013. 220 p.

14. CFF. Conselho Federal de Farmácia. A assistência farmacêutica no SUS. Comissão de Assistência Farmacêutica do Serviço Público do CRF-PR. - Brasília: Conselho Federal de Farmácia, 2010.

15. Monteguti BR, Diehl EE. O ensino de farmácia no sul do Brasil: preparando farmacêuticos para o sistema único de saúde? Trab. Educ. Saúde 2016; 14(1): 77-95. DOI: 10.1590/1981-7746-sol00008

16. Nakamura CA, Leite SN. A construção do processo de trabalho no Núcleo de Apoio à Saúde da Família: a experiência dos farmacêuticos em um município do sul do Brasil. Ciência Saúde Col. 2016; 21(5): 565-1572. DOI: 10.1590/1413-81232015215.17412014

17. Vieira MRS, Lorandi PA, Bousquat A. Avaliação da assistência farmacêutica à gestante na rede básica de saúde do Município de Praia Grande, São Paulo, Brasil. Cad. Saúde Púb. 2008; 24(6): 1419-1428.

18. Alencar TOS, Nascimento MAA, Alencar BR. Assistência farmacêutica na estratégia saúde da família: uma análise sobre o acesso. Rev. Bras. Farm. 2013; 94(3): 219-226.

19. Vieira AMP, Cruz APF, Cunha VF. Assistência farmacêutica em Unidades básicas de saúde do Município de Parnamirim - RN. Rev Cient Escola da Saúde. 2013; 2(1): 35-49.

20. Silva HOV, Quintal JS, Queiroz LMD. Caracterização da unidade básica de saúde do Guamá: cenário de prática do observatório farmacêutico da Faculdade de Ciências Farmacêticas, da Universidade Federal do Pará. Anais do III Congresso de Educação em Saúde da Amazônia (COESA), Universidade Federal do Pará - 12 a 14 de novembro de 2014. ISSN 2359-084X.

21. Busato MA, Lunkes EF. Assistência farmacêutica na estratégia saúde da família em um município de pequeno porte de Santa Catarina. Rev. Saúde Públ. Santa Cat., Florianópolis. 2012; 5(1): 8-18.

22. Prefeitura Municipal de Almirante Tamandaré. Secretaria Municipal de Saúde. Plano Municipal de Saúde 20142017. Almirante Tamandaré, 2013. 
23. Prefeitura Municipal de Arapoti. Secretaria Municipal de Saúde. Plano Municipal de Saúde 2014-2017. Arapoti, 2013.

24. Prefeitura Municipal de Campina do Simão. Secretaria Municipal de Saúde. Plano Plurianual de Saúde 20132017. Campina do Simão, 2013.

25. Prefeitura Municipal de Campo Magro. Secretaria Municipal de Saúde. Plano Municipal de Saúde 2014-2015. Campo Magro, 2013.

26. Prefeitura Municipal de Campo Mourão. Secretaria Municipal de Saúde. Plano Municipal de Saúde 2014-2017. Campo Mourão, 2014.

27. Prefeitura Municipal de Cascavel. Secretaria Municipal de Saúde. Plano Municipal de Saúde 2014-2017. Cascavel, 2013.

28. Prefeitura Municipal de Curitiba. Secretaria Municipal de Saúde. Plano Municipal de Saúde 2014-2017. Cascavel, 2013.

29. Prefeitura Municipal de Ibiporã. Secretaria Municipal de Saúde. Plano Municipal de Saúde 2013-2017. Ibiporã, 2013.

30. Prefeitura Municipal de Jacarezinho. Secretaria Municipal de Saúde. Plano Municipal de Saúde 2014-2017. Jacarezinho, 2013.

31. Prefeitura Municipal de Lapa. Secretaria Municipal de Saúde. Plano Municipal de Saúde 2014-2017. Lapa, 2013.

32. Prefeitura Municipal de Londrina. Secretaria Municipal de Saúde. Plano Municipal de Saúde 2014-2017. Londrina, 2013.

33. Prefeitura Municipal de Maringá. Secretaria Municipal de Saúde. Plano Municipal de Saúde 2014-2017. Maringá, 2013.

34. Prefeitura Municipal de Pinhais. Secretaria Municipal de Saúde. Plano Municipal de Saúde 2014-2017. Pinhais, 2013.

35. Prefeitura Municipal de Ponta Grossa. Secretaria Municipal de Saúde. Plano Municipal de Saúde 2014-2017. Ponta Grossa, 2013.
36. Prefeitura Municipal de São José dos Pinhais. Secretaria Municipal de Saúde. Plano Municipal de Saúde 20142017. São José dos Pinhais, 2014.

37. Prefeitura Municipal de Tupãssi. Secretaria Municipal de Saúde. Plano Municipal das Ações para o Desenvolvimento dos Trabalhos Voltados a Saúde no Município de Tupãssi. Tupãssi, 2010.

38. Prefeitura Municipal de Antonina. Secretaria Municipal de Saúde. Plano Municipal de Saúde 2014-2017. Antonina, 2013.

39. Prefeitura Municipal de Balsa Nova. Secretaria Municipal de Saúde. Plano Municipal de Saúde 2016-2019. Balsa Nova, 2015.

40. Prefeitura Municipal de Califórnia. Secretaria Municipal de Saúde. Plano Municipal de Saúde 2014-2017. Califórnia, 2015.

41. Prefeitura Municipal de Cambará. Secretaria Municipal de Saúde. Plano Municipal de Saúde 2014-2017. Cambará, 2013.

42. Prefeitura Municipal de Carambeí. Secretaria Municipal de Saúde. Plano Municipal de Saúde 2014-2017. Carambeí, 2013.

43. Prefeitura Municipal de Jaguariaíva. Secretaria Municipal de Saúde. Plano Municipal de Saúde 2014-2017. Jaguariaíva, 2013.

44. Prefeitura Municipal de Laranjeiras do Sul. Secretaria Municipal de Saúde. Plano Municipal de Saúde 20142017. Laranjeiras do Sul, 2015.

45. Prefeitura Municipal de Rebouças. Secretaria Municipal de Saúde. Plano Municipal de Saúde 2014-2017. Rebouças, 2013.

46. Prefeitura Municipal de Santa Fé. Secretaria Municipal de Saúde. Plano Municipal de Saúde 2014-2017. Santa Fé, 2013.

47. Prefeitura Municipal de Vera Cruz do Oeste. Secretaria Municipal de Saúde. Plano Municipal de Saúde 20142017. Vera Cruz do Oeste, 2013. 